\title{
Correction and simplification to "The order of a stabilizing regulator is sufficient a priori information for adaptive stabilization"
}

\author{
Bengt Mårtensson \\ Institute for Dynamical Systems, University of Bremen, P.O. Box \\ 330 440, D-2800 Bremen, Germany
}

\section{Jan Willem Polderman}

Department of Applied Mathematics, University of Twente, P.O. Box 217, 7500 AE Enschede, The Netherlands

Received 14 August 1992

Revised 2 December 1992

Abstract: This note corrects a mistake in a paper by Mårtensson (1986). The main conclusion there (as reflected in the title) remains unchanged, only the construction of the 'universal controller' has to be carried out slightly differently.

Keywords: Adaptive Stabilization; universal controllers; adaptive control.

\section{Introduction}

This note corrects a mistake in [6]. The main conclusion there (as reflected in the title) remains unchanged; only the construction of the 'universal controller' has to be carried out slightly differently. The note also corrects 'isomorphic' mistakes in $[7,8]$, which also gave a discrete-time version of the result in [6].

In [6] an adaptive controller was presented, claimed to stabilize any multivariable linear, timeinvariant, finite-dimensional plant for which the order $l$ of some (linear, time-invariant) controller was known. The construction was based on two parts: Constructing a smooth, dense curve in controller space (viewed as the $M \times P$-matrices), and establishing that the adaptation 'works', i.e.

Correspondence to: J.W. Polderman, Department of Applied Mathematics, University of Twente, P.O. Box 217, 7500 AE Enschede, The Netherlands that the states will asymptotically converge to 0 , while the parameter $k$ converges to a finite value. Unfortunately, the first part was in error. The sentence 'It follows from (6)-(9) that $\{g(h) N(h)$, $h=h(k), k \in \mathbb{R}\}$ is a dense subset of the space of $M \times P$ matrices' is not correct. For the 'proof' of that statement (carried out in more detail in [8]), the following, erroneous, 'property' of almost periodic functions $N: \mathbb{R} \rightarrow S^{M P-1}$ was used: For any open set $O \subset S^{M P-1}$ there is a sequence of equidistant intervals $I_{v}$, with length bounded from below, such that $N\left(I_{v}\right) \subset O$ for all $v$. This property does not follow from the definition of almost periodicity, and a counterexample can easily be constructed.

It is unknown to the authors whether the proof in [6] can be fixed or not. Instead, another construction, which is much more natural and simple, will be presented. It is based upon using a dense curve directly, instead of the unnatural approach in [6], using a decomposition in 'size' and 'direction'.

The present note has been written to be selfcontained in the sense that it contains a full proof on the main result, without reference to [6].

Throughout the paper, $\|\cdot\|$ denotes the Euclidean norm of $\mathbb{R}^{n}, n$ being clear from the context. We will also talk about $\mathscr{L}_{2}$ spaces without specifying the domain and co-domain.

\section{The adaptive control problem}

The plant is assumed to be representable by equations of the form

$$
\begin{aligned}
& \dot{x}(t)=A x(t)+B u(t), \quad x(t) \in \mathbb{R}^{n}, u(t) \in \mathbb{R}^{m}, \\
& y(t)=C x(t), \quad y(t) \in \mathbb{R}^{m} .
\end{aligned}
$$

The order $n$ and the matrices $A, B, C$ are not assumed to be known. We only make the following assumption on a priori knowledge about (2.1). 
Assumption 2.1. An integer $l$ is known such that there exists an asymptotically stabilizing compensator for (2.1) of the form

$\dot{z}(t)=F z(t)+G y(t), \quad z(t) \in \mathbb{R}^{l}$,

$u(t)=H z(t)+K y(t)$.

We note the following lemma, which is proved in $[4$, Theorem 7].

Lemma 2.2. Assumption 2.1 implies that the pair $(A, B)$ is stabilizable and the pair $(C, A)$ detectable.

Note that some forms of a priori knowledge translates directly to the knowledge of such an $l$ : for example, if $n$ were known, we could take $l=n$; if $\operatorname{det} C B \neq 0$ and (2.1) is minimum-phase then $l=0$ would be possible, etc.

With $l$ at our disposal, we can conceptually convert the original problem to the problem of stabilizing with static gain feedback by considering the dynamical elements of the controller as belonging to the plant: Define

$\tilde{x}=\left[\begin{array}{l}x \\ z\end{array}\right], \quad \tilde{u}=\left[\begin{array}{c}u \\ \dot{z}\end{array}\right], \quad \tilde{y}=\left[\begin{array}{l}y \\ z\end{array}\right]$

and

$\tilde{A}=\left[\begin{array}{cc}A & 0 \\ 0 & 0\end{array}\right], \quad \tilde{B}=\left[\begin{array}{cc}B & 0 \\ 0 & I\end{array}\right]$,

$\tilde{C}=\left[\begin{array}{ll}C & 0 \\ 0 & I\end{array}\right], \quad \tilde{K}=\left[\begin{array}{ll}K & H \\ G & F\end{array}\right]$.

Furthermore, define $M=m+l$ and $P=p+l$. The closed-loop system (2.1)-(2.2) can now be written as

$\dot{\tilde{x}}(t)=\tilde{A} \tilde{x}(t)+\tilde{B} \tilde{u}(t)$,

$\tilde{y}(t)=\tilde{C} \tilde{x}(t)$,

$\tilde{u}(t)=\tilde{K} \tilde{y}(t)$.

Note that since $(A, B)$ is stabilizable $((C, A)$ is detectable), so is $(\tilde{A}, \tilde{B})((\tilde{C}, \tilde{A}))$.

Rephrased in terms of the representation (2.3), Assumption 2.1 states that there exists some, in general not known, matrix $\tilde{K}_{0} \in \mathbb{R}^{M \times P}$ such that $\tilde{u}=\tilde{K}_{0} \tilde{y}$ stabilizes the plant, i.e. such that $\tilde{A}+\tilde{B} \tilde{K}_{0} \tilde{C}$ has all its eigenvalues in the open lefthalf plane.

The adaptive algorithm is based on a dense search through the controller space. The search path will be designed off-line and does not depend on the system or the data. However, the way in which the search path will be traversed will be determined by the input-output data.

The adaptive controller will be composed of two functions, $\tilde{K}: \mathbb{R}^{+} \rightarrow \mathbb{R}^{M \times P}$, and $h: \mathbb{R}^{+} \rightarrow \mathbb{R}$ satisfying the following assumptions:

(I) The 'controller curve' $\tilde{K}$ is Lipschitz-continuous. (Recall the definition: for every compact subset $\mathscr{K} \subset \mathbb{R}^{M \times P}$ there is a constant $\kappa$ such that, for $x, y \in \mathscr{K},\|\tilde{K}(x-y)\| \leq \kappa\|x-y\|$.)

(II) For all $a \in \mathbb{R}^{+}$, the image $\tilde{K}([a, \infty))$ is dense in $\mathbb{R}^{M \times P}$.

(III) The function $h$ is smooth, monotone increasing, with $\lim _{k \rightarrow \infty} h(k)=\infty$.

(IV) $\lim _{k \rightarrow \infty} k \frac{\mathrm{d} h}{\mathrm{~d} k}(k)=0$.

One possible $h$ is given by $h(k)=\sqrt{\log k}($ for $k>1)$. In Section 4 , examples of functions $\tilde{K}$ which satisfy these assumptions will be presented. Also note that it is enough to show (II) for one $a$, say $a=0$.

The adaptive controller is now given by

$$
\begin{aligned}
& \tilde{u}(t)=\tilde{K}(h(k(t))) \tilde{y}(t), \\
& \dot{k}(t)=\|\tilde{y}(t)\|^{2}+\|\tilde{u}(t)\|^{2} .
\end{aligned}
$$

The following result is standard, see e.g. [2].

Proposition 2.3. For all initial conditions $(\tilde{x}(0), k(0))$ there exists exactly one solution $(\tilde{x}, k)$ of equations $(2.3 a),(2.3 b)$ and $(2.4)$ defined on a maximal interval of existence $\left[0, t^{\dagger}\right)$. If $t^{\dagger}<\infty$, then $\lim _{t \rightarrow t^{\dagger}}\|(\tilde{x}, k)\|$ $=\infty$.

\section{The main result}

Theorem 3.1. Consider the system defined by equations (2.3a), (2.3b) and (2.4). Suppose that Assumption 2.1 holds, and that (I)-(IV) are satisfied. Then, for all initial condition $(x(0), z(0), k(0))$, it holds that $t^{\dagger}=\infty$, and

$\lim _{t \rightarrow \infty} k(t)=k_{\infty}<\infty, \quad \lim _{t \rightarrow \infty} x(t)=0, \quad \lim _{t \rightarrow \infty} z(t)=0$.

Before we prove Theorem 3.1 we will prove a simple lemma.

Lemma 3.2. Consider the system

$$
\begin{aligned}
& \dot{x}(t)=A x(t)+B u(t), \\
& y(t)=C x(t)
\end{aligned}
$$


and assume that $(C, A)$ is detectable. Then there exists constants $c_{0}, c_{1} \in \mathbb{R}$ such that for all $u(\cdot)$ and for all $t_{0}, t \in \mathbb{R}^{+}$it holds that

$$
\begin{aligned}
\|x(t)\|^{2} \leq & c_{0}\left\|x\left(t_{0}\right)\right\|^{2} \\
& +c_{1} \int_{t_{0}}^{t}\|u(\tau)\|^{2}+\|y(\tau)\|^{2} \mathrm{~d} \tau .
\end{aligned}
$$

Proof. By assumption, there exists a matrix $L$ such that $A-L C$ is asymptotically stable. Since $\dot{x}=(A-L C) x+B u+L y$, it follows from the variation-of-constants formula that

$$
\begin{aligned}
x(t)= & \mathrm{e}^{(A-L C)\left(t-t_{0}\right)} x\left(t_{0}\right) \\
& +\int_{t_{0}}^{t} \mathrm{e}^{(A-L C)(t-\tau)}(B u(\tau)+C y(\tau)) \mathrm{d} \tau \\
= & : L_{1, t-t_{0}}\left(x\left(t_{0}\right)\right)+L_{2, t-t_{0}}(u(\cdot), y(\cdot) .
\end{aligned}
$$

Since $A-L C$ is asymptotically stable, $\left\{L_{1, t}\right\}$ and $\left\{L_{2, t}\right\}$ are families of uniformly bounded operators, we have

$$
\begin{aligned}
& \|x(t)\|^{2} \leq 2\left\|L_{1, t-t_{0}}\right\|^{2}\left\|x\left(t_{0}\right)\right\|^{2} \\
& +2\left\|L_{2, t-t_{0}}\right\|^{2} \int_{t_{0}}^{t}\|u(\tau)\|^{2}+\|y(\tau)\|^{2} \mathrm{~d} \tau \\
& \leq 2 \sup _{t}\left\|L_{1, t}\right\|^{2}\left\|x\left(t_{0}\right)\right\|^{2} \\
& +2 \sup _{t}\left\|L_{2, t}\right\|^{2} \int_{t_{0}}^{t}\|u(\tau)\|^{2} \\
& +\|y(\tau)\|^{2} \mathrm{~d} \tau \text {. }
\end{aligned}
$$

With $c_{0}=2 \sup _{t}\left\|L_{1, t}\right\|^{2}$ and $c_{1}=2 \sup _{t}\left\|L_{2}, t\right\|^{2}$ is the desired inequality.

Proof of Theorem 3.1. The steps of the proof are as follows: Let $t^{\dagger}$ be as in Proposition 2.3. First, we show that if $k(t)$ remains bounded, then $t^{\dagger}=\infty$. The main work is now to show that if $k$ grows unbounded as $t \rightarrow t^{\dagger}$ then we arrive at a contradiction. For this, we establish the existence of 'nice' intervals of controllers. We estimate the possible growth within such an interval. Combining this with a general estimate of the norm of the state (Lemma 3.2), we get the contradiction. Finally, we show that the boundedness of $k$ implies the last two assertions.

If $t^{\dagger}<\infty$, it follows from Proposition 2.3 and Lemma 3.2 that $\lim _{t \rightarrow t^{+}} k(t)=\infty$. By $(2.4 \mathrm{~b}), k$ is monotonically nondecreasing; hence, $k$ either converges (in which case we may conclude that $t^{\dagger}=\infty$ ) or grows unbounded. Suppose that $k$ does not converge (do not assume that $t^{\dagger}=\infty$ ). We will derive a contradiction, thus proving the first two statements of the theorem.

By Assumption 2.1 there exists a $\tilde{K}_{0} \in \mathbb{R}^{M \times P}$ such that $\tilde{A}+\tilde{B} \tilde{K}_{0} \tilde{C}$ is asymptotically stable. Let $P_{0}=P_{0}^{\mathrm{T}}>0$ be the unique positive-definite solution of

$\left(\tilde{A}+\tilde{B} \tilde{K}_{0} \tilde{C}\right)^{\mathrm{T}} P_{0}+P_{0}\left(\tilde{A}+\tilde{B} \tilde{K}_{0} \tilde{C}\right)=-I$.

There exists a bounded open neighborhood $\Omega$ of $\tilde{K}_{0}$, such that for all $\tilde{K} \in \Omega$,

$(\tilde{A}+\tilde{B} \tilde{K} \tilde{C})^{T} P_{0}+P_{0}(\tilde{A}+\tilde{B} \tilde{K} \tilde{C}) \leq-\frac{1}{2} I$.

From (II) it follows that there exist countably many disjoint intervals $I_{j}=:\left(\alpha_{j}, \beta_{j}\right)(j=1,2, \ldots)$ such that $\tilde{K}\left(I_{j}\right) \subset \Omega$. By (I) we may conclude that $\beta_{j}-\alpha_{j} \geq \delta$ for some $\delta>0$.

The assumption of $k$ diverging implies that all intervals $I_{j}$ will be visited (also if $t^{\dagger}<\infty$ ). We will now estimate how much $h$ can grow after having entered $I_{j}$. We will see that for $j$ sufficiently large, $h$ cannot grow with the amount $\delta$, which is necessary to leave $I_{j}$, which will yield the contradiction.

Let $t_{j}$ be such that $h\left(k\left(t_{j}\right)\right)=\alpha_{j}$. We have

$$
\begin{aligned}
h(k(t))-\alpha_{j} & =\int_{t_{j}}^{t} \frac{\mathrm{d}}{\mathrm{d} \tau}(h(k(\tau)) \mathrm{d} \tau \\
& =\int_{t_{j}}^{t} \frac{\mathrm{d} h}{\mathrm{~d} k}(k(\tau)) \dot{k}(\tau) \mathrm{d} \tau \\
& =\int_{t_{j}}^{t} \frac{\mathrm{d} h}{\mathrm{~d} k}(k(\tau))\left(\|\tilde{u}(\tau)\|^{2}+\|\tilde{y}(\tau)\|^{2}\right) \mathrm{d} \tau \\
& \leq \gamma \int_{t_{j}}^{t} \frac{\mathrm{d} h}{\mathrm{~d} k}(k(\tau))\|\tilde{y}(\tau)\|^{2} \mathrm{~d} \tau
\end{aligned}
$$

(the last step used (2.4a)), with $\gamma=\sup _{K \in \Omega}$ $\|K+I\|^{2}<\infty$. Now assume that $t>t_{j}$ is such that $h\left(\left(t_{j}, t\right)\right) \subset I_{j}$. Under this assumption, it follows from (3.3) that $\frac{1}{2} x^{\mathrm{T}} P_{0 x}$ is an exponentially decaying Lyapunov function, as long as $h \in \Omega$. Therefore, for some $d$ (not depending on $j$ ) it holds that

$$
\int_{t_{j}}^{t}\|\tilde{y}(\tau)\|^{2} d \tau \leq d\left\|\tilde{x}\left(t_{j}\right)\right\|^{2}
$$


Combining this with Lemma 3.2 yields

$$
\begin{aligned}
& \int_{t_{j}}^{t}\|\tilde{y}(\tau)\|^{2} \mathrm{~d} \tau \\
& \quad \leq d\left(c_{0}\|\tilde{x}(0)\|^{2}+c_{1} \int_{0}^{t}\|\tilde{u}(\tau)\|^{2}+\|\tilde{y}(\tau)\|^{2} d \tau\right) \\
& \quad=\mathrm{d}\left(c_{0}\|\tilde{x}(0)\|^{2}+c_{1}\left(k\left(t_{j}\right)-k(0)\right)\right) .
\end{aligned}
$$

Inserting into (3.4) we get

$h(k(t))-\alpha_{j} \leq\left(c_{0}^{\prime}+c_{1}^{\prime} k\left(t_{j}\right)\right) \sup _{k, h(k) \in I_{j}} \frac{\mathrm{d} h}{\mathrm{~d} k}(k)$,

where $c_{0}^{\prime}=d \gamma\left(c_{0}\|\tilde{x}(0)\|^{2}-k(0)\right)$ and $c_{1}^{\prime}=d \gamma c_{1}$ depend on system constants and initial conditions, but not on $j$. For $j$ large, it holds that $\left(c_{0}^{\prime}+\right.$ $\left.c_{1}^{\prime} k\left(t_{j}\right)\right) \leq 2 c_{1}^{\prime} k\left(t_{j}\right)$, and, since $k\left(t_{j}\right)$ is less than or equal to any element in the set over which the supremum in (3.5) is taken, it holds that

$h(k(t))-\alpha_{j} \leq 2 c_{1}^{\prime} \sup _{k, h(k) \in I_{j}}\left(k \frac{\mathrm{d} h}{\mathrm{~d} k}(k)\right)$.

We may now conclude from (IV) that the righthand side of (3.6) goes to 0 when $j$ goes to infinity. In particular, eventually $h(k(t))-\alpha_{j}<\delta$. This establishes the contradiction.

We conclude that $k$ has a limit, say $\lim _{t \rightarrow \infty} k(t)$ $=k_{\infty}<\infty$. It remains to prove the last two assertions of the theorem, or equivalently, that $\lim _{t \rightarrow \infty} \tilde{x}(t)=0$.

By $(2.4 \mathrm{~b})$ it follows that $\tilde{u}, \tilde{y} \in \mathscr{L}_{2}$. Select $\tilde{L}$ so that $A-\tilde{L} \tilde{C}$ has all eigenvalues in the open left-half plane. Since $\dot{\tilde{x}}=(\tilde{\tilde{A}}-\tilde{L} \tilde{C}) \tilde{x}+\tilde{B} \tilde{u}+\tilde{L} \tilde{C} \tilde{y}$, we may consider $\tilde{x}(\cdot)$ as the image of $\tilde{y}(\cdot)$ and $\tilde{u}(\cdot)$ under a bounded affine operator between suitable $\mathscr{L}_{2}$ spaces. It follows that $\tilde{x}(\cdot) \in \mathscr{L}_{2}$. Since $k(\cdot)$ is bounded, we may conclude from (2.3a), (2.3b), and (2.4b) that also $\dot{\tilde{x}} \in \mathscr{L}_{2}$. It now follows that $\lim \tilde{x}(t)=0$ : first note that by considering every component individually, we may assume that $\tilde{x}$ is scalar. According to Hölder, $\dot{\tilde{x}} \tilde{x}$ is integrable, i.e.

$2 \int_{0}^{t} \dot{\tilde{x}} \tilde{x} \mathrm{~d} \tau=: M_{t}$

has a finite limit, as $t \rightarrow \infty$. But

$M_{t}=\int_{0}^{t} \frac{\mathrm{d}}{\mathrm{d} t} \tilde{x}(\tau)^{2} \mathrm{~d} \tau=\tilde{x}(t)^{2}-\tilde{x}(0)^{2}$.
Since $M_{t}$ converges, $\tilde{x}(t)$ must converge to a constant $\tilde{x}_{\infty}$. But $\tilde{x}(\cdot) \in \mathscr{L}_{2}$, so $\tilde{x}_{\infty}=0$.

\section{Dense curves}

In order to show that the theorem in the previous section is not void, we have to demonstrate a construction satisfying (I) and (II). There are many ways of doing this, and we will present two different functions $\tilde{K}$ satisfying the conditions (I) and (II). The first example is based on the skew line on the torus, whereas the second example relies on an enumeration of $\mathbb{Q}^{N}$.

For ease of notation, with $N=M P$ we identify $\mathbb{R}^{M \times P}$ (the controller space) with $\mathbb{R}^{N}$ via the coordinates, and present dense curves on $\mathbb{R}^{N}$ instead.

\subsection{A differential-geometric approach}

We next present a construction based on mapping the skew line on the torus onto $\mathbb{R}^{N}$. It yields a very simple, explicit formula. It is only valid for $N \geq 2$. (For $N=1$ one possible choice is $\tilde{K}(h)=h \sin h$.)

Proposition 4.1 For $N \geq 2$, let $a_{1}, \ldots, a_{N} \in \mathbb{R}$ be linearly independent over $\mathbb{Q}$. Define the mapping $\tilde{K}: \mathbb{R}^{+} \rightarrow \mathbb{R}^{N}$ by

$$
\tilde{K}(h)=\left(\frac{1}{\sum_{j=1}^{N} \sin ^{2} a_{j} h}-1\right)\left[\begin{array}{c}
\sin a_{1} h \\
\vdots \\
\sin a_{N} h
\end{array}\right] .
$$

Then

(i) $\tilde{K}$ is well defined and $\mathscr{C}^{\infty}$ for all $h>0$,

(ii) the image of $\tilde{K}$ is dense in $\mathbb{R}^{N}$.

Note that (i) in particular implies (I). By the remark following (I)-(IV), (ii) implies (II).

As an example of linearly independent real numbers $a_{1}, \ldots, a_{N}$, we mention $a_{j}=\sqrt{p_{j}}$ where $p_{j}$ is the $j$ th prime.

Informally, the construction can be understood as follows. The mapping is the composition of the skew line on the $N$-dimensional torus with a surjective mapping from the torus (with a finite number of singularity points removed) to $\mathbb{R}^{N}$. The skew line is dense on the torus. A surjective 'blow-up' maps the skew line into smooth and 
dense curve in $\mathbb{R}^{N}$ traversed with bounded velocity on bounded subsets, provided that the singularities are avoided.

Proof. (i) We only need to prove that the denominator $\left(\Sigma \sin ^{2} a_{j} h\right)$ never vanishes for $h>0$. If this happens, say for $h=h_{0}>0$, then $a_{j} h_{0}=0 \bmod \pi$ for $j=1, \ldots, n$, which implies that there exist $k_{1}, \ldots, k_{N} \in \mathbb{Z}^{+}$such that

$a_{j} h_{0}=k_{j} \pi, \quad j=1, \ldots, N$.

Let $p, q \in\{1, \ldots, n\}$ be distinct. Multiplying the $p$ th equation of (4.2) by $k_{q}$ and the $q$ th equation by $k_{q}$, subtracting, and finally dividing by $h_{0} \neq 0$, we get $k_{q} a_{p}-k_{p} a_{q}=0$. This contradicts the linear independent of the $a_{j}$ s s over $\mathbb{Q}$.

(ii) Denote the $N$-dimensional torus $\tau^{N}=\mathbb{R}^{N} /$ $2 \pi \mathbb{Z}$ by $T$ and the $N$-dimensional interval $[-1,1]^{N}$ by $I$. With $x=\left[x_{1}, \ldots, x_{N}\right]^{\mathrm{T}}$, define the mappings $\alpha: \mathbb{R}^{+} \rightarrow T, \beta: T \rightarrow I, \quad$ and $\quad \gamma: I \backslash\{0\} \rightarrow \mathbb{R}^{N}$ by $\alpha(h)=\left[a_{1} h \bmod 2 \pi, \ldots, a_{N} h \bmod 2 \pi\right]^{\mathrm{T}}, \quad \beta(x)=$ $\left[\sin x_{1}, \ldots, \sin x_{N}\right]^{\mathrm{T}}$, and $\gamma(x)=\left(1 / \sum_{j=1}^{N} x_{j}^{2}-1\right)$ $\left[x_{1}, \ldots, x_{N}\right]^{\mathrm{T}}$. By part (i), it follows that $\tilde{K}=\gamma \circ \beta \circ \alpha$.

It is well known that $\alpha$ has dense image (Kronecker's theorem, [3, Theorem 444], see also $[1$, Section 3.2]). Since $\gamma$ preserves rays (considering $I$ as a subset of $\left.\mathbb{R}^{N}\right)$, and since $\left(1 / \sum_{j=1}^{N} x_{j}^{2}-1\right)$ takes on all values in $[0, \infty)$, we see that $\gamma$ is surjective and continuous. Clearly, also $\beta$ is continuous and surjective. Since $\alpha$ has dense image, two applications of the following simple topological lemma completes the proof of (ii).

Lemma 4.2. Let $f: X \rightarrow Y$ be a surjective, continuous mapping between topological spaces $X$ and $Y$. For any dense $E \subset X$ it holds that $f(E)$ is dense in $Y$.

Proof. Assume that $f(E)$ is not dense in $Y$, i.e. that for some nonempty open set $O$ it holds that $O \cap f(E)=\emptyset$. Since $f$ is continuous, $f^{-1}(O)$ is open in $X$ and since $f$ is surjective, it is nonempty. From $O \cap f(E)=\emptyset$, we conclude that $f^{-1}(O) \cap E=\emptyset$. Thus, $E$ cannot be dense, which is a contradiction.

\subsection{A dense curve based on an enumeration of $\mathbb{Q}^{N}$}

A second example of a dense curve is obtained as follows.
Proposition 4.3. Let $\left\{q_{i}\right\}_{i \in \mathbb{N}}$ be an enumeration of $\mathbb{Q}^{N}$. Define $\left\{\tau_{i}\right\}$ by

$\tau_{0}=0$,

$\tau_{i+1}=\tau_{i}+\left\|q_{i+1}-q_{i}\right\|$ for $i>1$.

Define $\tilde{K}: \mathbb{R}^{+} \cup(0\} \rightarrow \mathbb{R}^{N}$ by

$\tilde{K}\left((1-\lambda) \tau_{i}+\lambda \tau_{i+1}\right):=(\lambda-1) q_{i}+\lambda q_{i+1}$,

$0 \leq \lambda<1$.

Then $\tilde{K}$ has properties (I) and (II).

In words: the image $\tilde{K}\left(\mathbb{R}^{+}\right)$is the linear interpolation of the 'enumeration' of $\mathbb{Q}^{N}$, traversed with constant speed. From the construction, it is obvious that $\tilde{K}$ satisfies the requirements, so the proof is omitted.

Remark 4.4. Given $h, \tilde{K}(h)$ can be calculated as follows. First determine the unique index $i$ such that $\tau_{i} \leq h<\tau_{i+1}$. Then define $\lambda=\left(h-\tau_{i}\right) /$ $\left(\tau_{i+1}-\tau_{i}\right)$, which is well defined since $\tau_{i+1} \neq \tau_{i}$. Finally, $\tilde{K}(h)=\lambda q_{i}+(1-\lambda) q_{i+1}$.

Remark 4.5. When trying to write an algorithm to enumerate $\mathbb{Q}^{N}$, one notes that the task is much simpler if we do not have to worry about repetitions in the sequence $q_{0}, q_{1}, \ldots$. Therefore, we remark that the requirement of $\left\{q_{i}\right\}_{i}$ to be an enumeration may be replaced with the weaker requirement that $q_{i}$ is a surjective mapping $\mathbb{N} \rightarrow \mathbb{Q}$ satisfying $q_{i} \neq q_{i+1}$.

Accepting the enumeration as given, the latter construction possibly provides an example which is easier to understand (and prove!). However, it does not yield such an explicit formula as the first construction. We remark that in [5] 'pseudo-code' implementations of algorithms similar to the one above was given. The pseudo-code algorithms there generalize in an obvious way to generate 'enumerations' of $\mathbb{Q}^{N}$ for arbitrary $N$.

Finally, we remark that the controller given by (2.4) and (4.1), with, e.g., $h=\log (k+2)$ and $a_{i}=\sqrt{p_{i}}$, is indeed an extremely simple controller, for example, in the sense of effort of implementing it in your favorite simulation program. This is not to say that we claim the quantitative performance is neither good nor acceptable, only to expose the fragility of some arguments comparing the 'simplicity' of different universal stabilizing 
algorithms. It is not clear if a construction containing elements like enumeration of the rationals qualifies as 'simple'.

\section{Acknowledgement}

The authors would like to thank Hartmut Logemann for many rewarding discussions.

\section{References}

[1] V.I. Arnold, Geometrical Methods in the Theory of Ordinary Differential Equations, vol. 250 (Springer, Berlin, 1983).

[2] J. Hale, Ordinary Differential Equations, (Robert E. Krieger Publ. Company, Revised ed., 1980).
[3] G.H. Hardy and E.M. Wright, An Introduction to the Theory of Numbers (Oxford University Press, Oxford 5th ed. 1985).

[4] M.L.J. Hautus, Stabilization, controllability, and observability of linear autonomous systems, Proc. Ned. Akad. Wetenschappen, Ser. A. 73, (1970) 448-455.

[5] H. Logemann and B. Mårtensson, Adaptive stabilization of infinite dimensional systems, IEEE Trans. Automat. Control, to appear.

[6] B. Mårtensson, The order of any stabilizing regulator is sufficient a priori information for adaptive stabilization, System Control Lett. 6 (1985) 87-91.

[7] B. Mårtensson, Adaptive stabilization of general, multivariable, continuous- or discrete-time systems, in: C.I. Byrnes and $\mathrm{A}$. Lindquist, eds., Modelling, Identification and Robust Control (North-Holland, Amsterdam 1986).

[8] B. Mårtensson, Adaptive stabilization, Ph.D. Thesis, Lund Institute of Technology, Department of Automatic Control. Lund, Sweden, 1986. 